\title{
Inversion of Radio Wave Absorption Data To Establish Ionospheric Properties. I. Nondeviative Absorption
}

\author{
Albert D. Wheelon \\ (May 15, 1963)
}

\begin{abstract}
Nondeviative absorption of high frequency radio waves which penetrate the lower ionospheric layers is analyzed in terms of the usual expression:

$$
\ln \left(\frac{P_{R}}{P_{0}}\right)=A(\omega)=\frac{4 \pi e^{2}}{m c} \int d s \frac{N \nu}{\left[\nu^{2}+\left(\omega \pm \omega_{L}\right)^{2}\right]} .
$$

Classical approximate predictions of the absorption function $A(\omega)$ for several ionospheric electron density $N$ and collision frequency $\nu$ models derived from this expression are first reviewed. It is then shown that by solving the above integral equation, one can establish an explicit relationship between $N$ and $\nu$ at any height from a measured knowledge of the absorption function $A(\omega)$ as a function of carrier frequency $\omega$. Thus, a knowledge of the electron density profile can be used to reconstruct the collision frequency profile and conversely. It is shown that the usual assumption $A(\omega)=A_{0} \omega^{-2}$ corresponds to an impossible ionospheric model. Several examples are given to illustrate the analytical inversion technique and to indicate its sensitivity to low frequency portions of the measured absorption function.
\end{abstract}

\section{Introduction}

The absorption of radio waves reflected from the ionosphere has been important for several decades in connection with estimating the required power margins of shortwave communication links. More recently, it has been recognized that the variation of radio wave absorption with carrier frequency and other independent propagation variables represents a valuable new tool for studying the electron density and/or collision frequency profile of the ionosphere [Little, 1961]. The general absorption problem divides itself naturally into two topics: deviative and nondeviative. The process is called deviative if the wave is reflected by the layer which is primarily responsible for the absorption. Deviative absorption is the more difficult of the two types, since one must then recognize both the absorption and refractive effects simultaneously at each point along the ray trajectory. Nondeviative absorption refers to wave absorption by layers which lie well below the reflecting layers; in this case the ray paths are neither bent nor "stretched" in the region of principal absorption. Of course, a radio star or satellite signal which passes completely through the ionosphere is also beset by nondeviative absorption. Figure 1 illustrates these three cases.

It is commonly held that the $D$ region of the ionosphere between 70 and $80 \mathrm{~km}$ is primarily responsible for shortwave absorption. The absorption per unit length is roughly proportional to the product of electron density $N$ and collision frequency $\nu$, and the $D$ region is that in which the suddenly rising electron density profile and exponentially declining collision frequency have the greatest product. For vertical incidence, the deviative-to-nondeviative frequency "watershed" occurs at several megacycles, corresponding to the critical frequency of the $E$ region by day.

This first paper is concerned with nondeviative absorption, and is therefore relevant to both the absorption of radio star/satellite signals propagating through the ionosphere and $F$ layer reflection by day and night. We base our treatment here on the Appleton-Hartree expressions for the electromagnetic response of an ionospheric plasma which suffers collisions with the neutral particles in a way which

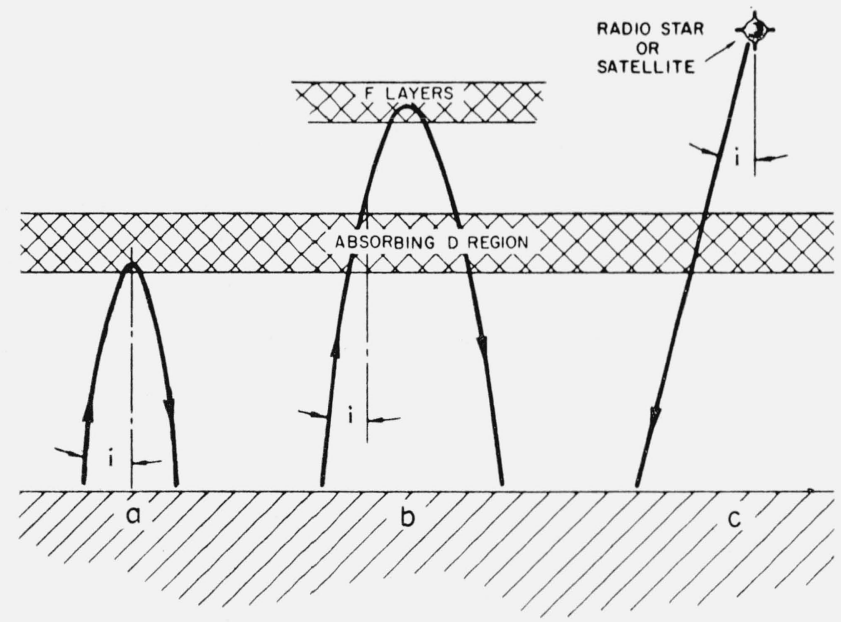

FIGURE 1. Typical ray paths for radiowave absorption for rays entering the absorbing D-region with an angle of incidence $i$ : (a) deviative absorption in reflection region; (b) nondeviative absorption below reflecting $\mathrm{F}$ layers, and (c) nondeviative absorption of a radio star or satellite signal. 
does not depend on the energy of the electrons themselves. We shall return in a later paper to consider the effect of modifications of this analysis called out by the revision of the Appleton-Hartree formula which recognizes an electron energy dependence of the collision cross section with neutral atoms [Sen and Wyller, 1960].

The starting point for this analysis is the magnetoionic expression for field strength attenuation [Ratcliffe, 1959]. Above several megacycles, it is probably safe to use the quasi-longitudinal approximation, which relates the received field strength $E_{R}(\omega)$ to that which would be received if there were no absorption $E_{0}(\omega)$ by the following expression:

$$
E_{R}(\omega)=E_{0}(\omega) \exp -\frac{2 \pi e^{2}}{m c} \int_{\text {path }} d s \frac{N \nu}{\nu^{2}+\left(\omega \pm \omega_{L}\right)^{2}} \frac{1}{\mu}
$$

where

$e=4.8 \times 10^{-10}$ esu, electronic charge.

$m=9.11 \times 10^{-28} \mathrm{~g}$, electronic mass.

$c=3 \times 10^{10} \mathrm{~cm} / \mathrm{sec}$, speed of light.

$\omega_{L}=$ Local gyrofrequency of the earth's magnetic field times the cosine of the angle $\psi$ between the local ray direction and the magnetic field vector.

$\nu=$ Local collision frequency.

$N=$ Local electron density.

$\mu=$ Corresponding local refractive index.

The integral in (1.1) is to be taken along the nominal ray path determined by Snell's law and the refractive index profile $\mu(y)$. For the nondeviative case under consideration, the refractive index may be set equal to unity, and the integral along the ray path in the absorbing region replaced by that along a rectilinear path,

$$
d s=d y \sec i
$$

where $i$ is the angle of inclination of the ray with respect to the local vertical $(y)$ as it traverses the absorbing region (see fig. 1). This allows (1.1) to be rewritten as:

$E_{R}(\omega)=E_{0}(\omega) \exp -\frac{2 \pi e^{2}}{m c} \sec i \int_{0}^{\infty} d y \frac{N(y) \nu(y)}{\nu^{2}(y)+\left(\omega \pm \omega_{L}\right)^{2}}$.

The height range of integration here has been extended from ground level to infinity, since the nondeviative absorption is confined to the lower reaches of the ionosphere and negligible error is introduced by extending the integral indefinitely above this. Strictly speaking, expression (1.2) corresponds to the radio star/satellite case shown in figure 1 and one should insert an additional factor of two in the exponent to account for the double ionospheric traverse associated with $F$ layer reflection.

\section{Classical Predictions of Absorption}

Classical predictive analysis of nondeviative absorption approximates the integral term in (1.2) by assuming that the carrier frequency of the wave is considerably greater than the collision frequency over the height range for which the product $N \nu$ is significantly large. The same assumption implies that $\omega \gg \omega_{L}$, and for $F$ layer (reflective) propagation one obtains:

$$
E_{R}(\omega)=E_{0}(\omega) \exp -\left(\frac{4 \pi e^{2}}{m c}\right) \sec i \frac{1}{\omega^{2}} \int_{0}^{\infty} d y N \nu .
$$

Since the secant of the angle of incidence is roughly proportional to the transmission distance $D$ for flat earth propagation via thin layers, one obtains the usual absorption expression [Best and Ratcliffe, 1938].

$$
E_{R}=E_{0} \in \exp -\eta D \lambda^{2} .
$$

This simple law has been confirmed approximately by numerous shortwave experiments [Farmer and Ratcliffe, 1935]. It is precisely this scaling law which encourages one to work as close as possible to the maximum usable frequency (MUF) on any given day, so as to reduce the effects of nondeviative absorption.

A significant diurnal variation of the measured exponential coefficient $\eta$ in $(2.2)$ is properly attributed to the corresponding diurnal variability of the electron density $N$ in the defining integral (2.1). This variability was calculated theoretically by Appleton [1937] using the Chapman model for the electron density versus height profile [Chapman, 1931].

$$
N(y, \chi)=N_{0} \exp -\frac{1}{2}\left(\frac{y}{H}+A \rho_{0} H \sec \chi e^{-y / H}\right)
$$

where $\chi$ is the time-variable solar zenith angle, $H$ the scale height of the exponential atmosphere assumed, $A$ the photo-absorption coefficient, and $\rho_{0}$ the sea level neutral particle density. Appleton evaluated the diurnal variability of the absorption factor by combining (2.3) with an exponential collision frequency profile ${ }^{1} \nu(y)=\nu_{0} e^{-y / H}$. Carrying out the integral in (2.1) Appleton [6] found

$$
E_{R} \simeq E_{0} \exp -\eta^{\prime} D \cos ^{3 / 2} \chi \lambda^{2} .
$$

A good deal of effort has recently been devoted to comparing accumulating experimental data with expression (2.4). This effort has been moderately successful and many workers have been content to let the matter rest there. On the other hand, the extensive NBS empirical studies favor a linear variation of the absorption factor ${ }^{2}$ with $\cos \chi$, rather than the $\cos ^{3 / 2} \chi$ term in (2.4). Beynon and Davies [1954] found that a frequency and zenith angle dependent coefficient of $\lambda^{2}, A+B F(f) \cos \chi$, gives a better overall fit to the experimental data gathered on $2.0 \mathrm{Mc} / \mathrm{s}$ in England. Davies and Hagg [1955] have even challenged the $\lambda^{2}$ scaling law of (2.4) after careful examination of absorption data on several

\footnotetext{
1 Which is also implicit in the Chapman model of (2.3).

${ }^{2}$ Ionospheric radio propagation (June 1948), NBS Cire. 462, 37-38; 112-113.
} 
frequencies at high latitudes (Prince Rupert), with corresponding variation in the exponent in $\cos ^{m} \chi$ between $m=0.29$ and 0.82 . Chapman and Davies [1958] have also commented on the approximate constancy of daytime absorption.

This variability and/or uncertainty probably represents a fundamental deficiency in the simplified prediction (2.4). This deficiency can be traced, at least in part, to the unwarranted simplification of the original expression (1.2). The assumption that "the collision frequency $\nu$ is small compared with the carrier frequency $\omega$ in those regions where $N_{\nu}$ is large," is a qualitative argument whose precision and value has lost ground as more accurate models of the lower ionosphere have become available. Another way of looking at the matter of predominant absorption heights is to note that the factor

$$
\frac{\nu}{\nu^{2}+\omega^{2}}
$$

has its maximum value at the height corresponding to $\nu=\omega$, and so long as $N$ has a respectable value at such heights (i.e., the $D$ region for $H F$ ), the frequency variable denominator in (1.2) ought not to be approximated to the form of (2.1). In point of fact, it is just this denominator - in a delicate balance-which can account for the spectrum of frequency and zenith angle scaling law exponents observed experimentally.

Jaeger [1947] published an important analysis of nondeviative absorption based on the complete absorption integral in (1.2) which demonstrates this natural variability. His calculation is based on the Chapman electron density model (2.3) and an exponential collision frequency profile,

$$
E_{R} \simeq E_{0} \exp -\left[\eta D \cos ^{3 / 2} \chi \lambda^{2} J\left(\frac{\omega \sec \chi}{2 \nu_{0}}\right)\right]
$$

where

$$
\begin{aligned}
J(\beta)=4 \pi \beta^{3 / 2} \sqrt{e}\{\cos \beta[ & \left.\frac{1}{2}-C(\beta)\right] \\
& \left.+\sin \beta\left[\frac{1}{2}-S(\beta)\right]\right\}
\end{aligned}
$$

and

$$
\begin{aligned}
& C(x)=\frac{1}{\sqrt{2 \pi}} \int_{0}^{x} d t \frac{\cos t}{\sqrt{t}} \\
& S(x)=\frac{1}{\sqrt{2 \pi}} \int_{0}^{x} d t \frac{\sin t}{\sqrt{t}} .
\end{aligned}
$$

The function $J(\beta)$ ranges from 0 to 4.133 as $\beta$ varies from zero to infinity. The small argument behavior of this function,

$$
\lim _{\beta \rightarrow 0} J(\beta) \simeq 2 \pi \sqrt{e} \beta^{3 / 2}
$$

indicates that the absorption exponent is independent of solar zenith angle (i.e., time) for small $\beta$. This behavior thus provides a flexible range of frequency and zenith angle scaling law exponents,

$$
\lambda^{1 / 2} D \text { to } \lambda^{2} D \cos ^{3 / 2} \chi
$$

as suggested by experiment. However, it does not yet seem that this broader theory has been put to the test of close comparison with experimental data.

The real point of the matter would seem to be that theory has reached the point of diminishing returns in fitting theoretical electron density and collision frequency profiles to experimental absorption data, either through the complete magnetoionic expression (2.4) or its approximate form (2.1). The present troubles run sufficiently deep to encourage one to take another approach to the entire problem and provide the motivation of the present series of papers.

In this first paper we shall regard absorption measurements as a means of establishing the electron density profile, rather than as a means of verifying preconceived notions of how this profile ought to look. In this sense, we shall regard (1.2) as an integral equation for finding $N(y)$ from a measured knowledge of $E_{R} / E_{0}$ as a function of carrier frequency. We shall show that this integral equation can be solved explicitly, thereby circumventing the need for fitting adjustable parameters in theoretical profile models.

\section{Inversion of the Absorption Integral}

Let us now turn to the direct problem of recovering the electron density or collision frequency profile from a measured variation of absorption with carrier frequency. Actually, we shall find a relation between electron density and collision frequency at any height as the solution of the integral equation:

where

$$
A(\omega)=\int_{0}^{\infty} d y \frac{N(y) \nu(y)}{\nu^{2}(y)+\left(\omega \pm \omega_{L}\right)^{2}}
$$

$$
A(\omega)=\frac{-m c}{4 \pi e^{2}} \cos i \log _{\epsilon}\left[\frac{E_{R}(\omega)}{E_{0}(\omega)}\right]
$$

is related to the basic measurements of field strength. Let us assume that the function $A(\omega)$ is measured by carefully calibrated experiments and show how one can solve the integral (3.1).

The first step is to convert the integral in (3.1) over all height ranges $(0<y<\infty)$ to an integral over the collision frequency $\nu$ between corresponding limits. The value of $\nu$ for an infinite height above the earth is zero, since the atmosphere constituents with which the electrons make collisions decay rapidly with altitude. Even in the case of $F$ layer propagation (fig. 1b), the collision frequency at the top of the ray path is probably $10^{3} \mathrm{c} / \mathrm{s}$, and therefore several orders of magnitude smaller than the megacycle values for $\nu$ found in the absorbing $(D)$ region. The ground level value of collision frequency is very much greater than any carrier frequency (i.e., $\left.10^{23}\right)$ and is therefore reasonably taken to be infinity. These limit considerations are crucial to what fol- 
lows, but appear to be amply justified by the physics of the ionosphere as we know it today. With this change of independent variable, one is able to write,

$$
A(\omega)=\int_{0}^{\infty} d \nu \frac{\frac{\nu}{\nu^{\prime}} N(\nu)}{\nu^{2}+\left(\omega \pm \omega_{L}\right)^{2}} .
$$

Where the derivative of $\nu$ with respect to height is denoted by:

$$
\nu^{\prime}=\frac{d \nu}{d y}
$$

and $N$ is to be regarded as a function of collision frequency until the solution is effected. It seems that this trick requires that $N$ be a monotonic function of $\nu$, although closer analysis may supply a more generous restriction. We next note the following (Laplace transform) identity:

$$
\frac{\omega \pm \omega_{L}}{\nu^{2}+\left(\omega \pm \omega_{L}\right)^{2}}=\int_{0}^{\infty} d \eta e^{-\eta\left(\omega \pm \omega_{L}\right)} \cos (\eta \nu) .
$$

If this representation is substituted into (3.3) and the order of integration reversed, one finds

$$
\begin{gathered}
\left(\omega \pm \omega_{L}\right) A(\omega)=\int_{0}^{\infty} d \nu \frac{\nu}{\nu^{\prime}} N(\nu) \int_{0}^{\infty} d \eta e^{-\eta\left(\omega \pm \omega_{L}\right)} \cos (\eta \nu) \\
=\int_{0}^{\infty} d \eta e^{-\eta \omega}\left[e^{\mp \eta \omega_{L}} \int_{0}^{\infty} d \nu \cos (\eta \nu) \frac{\nu}{\nu^{\prime}} N(\nu)\right] .
\end{gathered}
$$

This reversal indicates that the unknown function of $\eta$ in square brackets is the Laplace transform of the known function $\left(\omega \pm \omega_{L}\right) A(\omega)$ on the left hand side of the equation. Inverting this relation, we find that

$$
e^{ \pm \eta \omega_{L}} \mathcal{L}^{-1}\left[\left(\omega \pm \omega_{L}\right) A(\omega)\right]=\int_{0}^{\infty} d \nu \cos (\nu \eta) \frac{\nu}{\nu^{\prime}} N(\nu),
$$

where $\mathcal{L}^{-1}[f]$ indicates the inverse Laplace transform of $f$. Equation (3.6) states that the Fourier cosine transform of the unknown function $\nu / \nu^{\prime} N(\nu)$ is equal to the known function on the left-hand side. Using the Fourier orthogonality result,

$$
\int_{0}^{\infty} d \eta \cos (a \eta) \cos (b \eta)=\pi \delta(a-b)
$$

where $\delta(x)$ is the singular Dirac delta function, one finds that

$$
\frac{\nu}{\nu^{\prime}} N(\nu)=\frac{1}{\pi} \int_{0}^{\infty} d x \cos (x \nu) e^{ \pm x \omega_{L}} \mathcal{L}^{-1}\left[\left(\omega \pm \omega_{L}\right) A(\omega)\right]_{x},
$$

where the inverse Laplace transform is to be expressed as a function of the dummy variable of integration $x$. Separating terms, we have the final solution

$$
N(y)=\frac{1}{\nu} \frac{d \nu}{d y} \frac{1}{\pi} \int_{0}^{\infty} d x \cos (x \nu) e^{ \pm x \omega_{L}} \mathcal{L}^{-1}\left[\left(\omega \pm \omega_{L}\right) A(\omega)\right]_{x}
$$

This equation provides an explicit relationship between the value of electron density $N$ at an arbitrary height $y$ and the collision frequency $\nu$ at the same height, and is therefore the desired solution. If the collision frequency profile is known as a function of height, this expression gives an explicit electron density profile. On the other hand, if it is the electron density profile which is known, one can reconstruct the collision frequency variation with height by integrating both sides of (3.8) from corresponding values of $y$ and $\nu$

$$
\begin{aligned}
\int_{y}^{\infty} d y N(y)= & -\int_{0}^{\nu(y)} d \nu \frac{1}{\nu} \frac{1}{\pi} \int_{0}^{\infty} d x \\
& \cos (\nu x) e^{ \pm x \omega_{L}} \mathcal{L}^{-1}\left[\left(\omega \pm \omega_{L}\right) A(\omega)\right]_{x} .
\end{aligned}
$$

This result completes the formal solution to the problem at hand.

\section{Illustrative Examples}

To show how the formal solution (3.8) can be used in a practical way to invert experimental measurements of the absorption function $A(\omega)$, it is instructive to consider several examples, in which we shall take $\omega_{L}=0$ for convenience. These examples will also indicate the extraordinary sensitivity of the electron density profile to the low frequency behavior of the absorption function.

A. Example 1. Let us consider first the logical consequences of the simplified classical absorption result [cf. (2.2)].

$$
A(\omega)=A_{0} \omega^{-2}
$$

which is plotted in figure 2. Noting that the inverse Laplace transform of $\omega^{-1}$ is just unity, we have

$$
\begin{aligned}
N(y) & =\frac{1}{\nu} \frac{d \nu}{d y} \frac{1}{\pi} \int_{0}^{\infty} d x \cos (\nu x) \mathcal{L}^{-1}\left(\frac{A_{0}}{\omega}\right)_{x} \\
& =A_{0} \frac{1}{\nu} \frac{d \nu}{d y} \frac{1}{\pi} \int_{0}^{\infty} d x \cos (\nu x) 1
\end{aligned}
$$

or using (3.7), we have:

$$
N(y)=A_{0} \frac{1}{\nu} \frac{d \nu}{d y} \delta(\nu-0)
$$

This result insists that the electron density be rigorously zero everywhere except at those (infinite) heights for which the collision frequency vanishes completely, where it must be infinite (see fig. 3a). Such a result is both ridiculous and inescapable, since it follows inevitably from the assumed frequency dependence (4.1) via the exact solution (3.8) of the absorption integral (3.1) describing the process. One can verify that (4.2) is the solution of (3.1) by substituting it therein and easily recovering the result (4.1).

The fault lies with the implicit assumption that(4.1) describes the frequency variation of the absorption factor for all frequencies. It certainly does not 


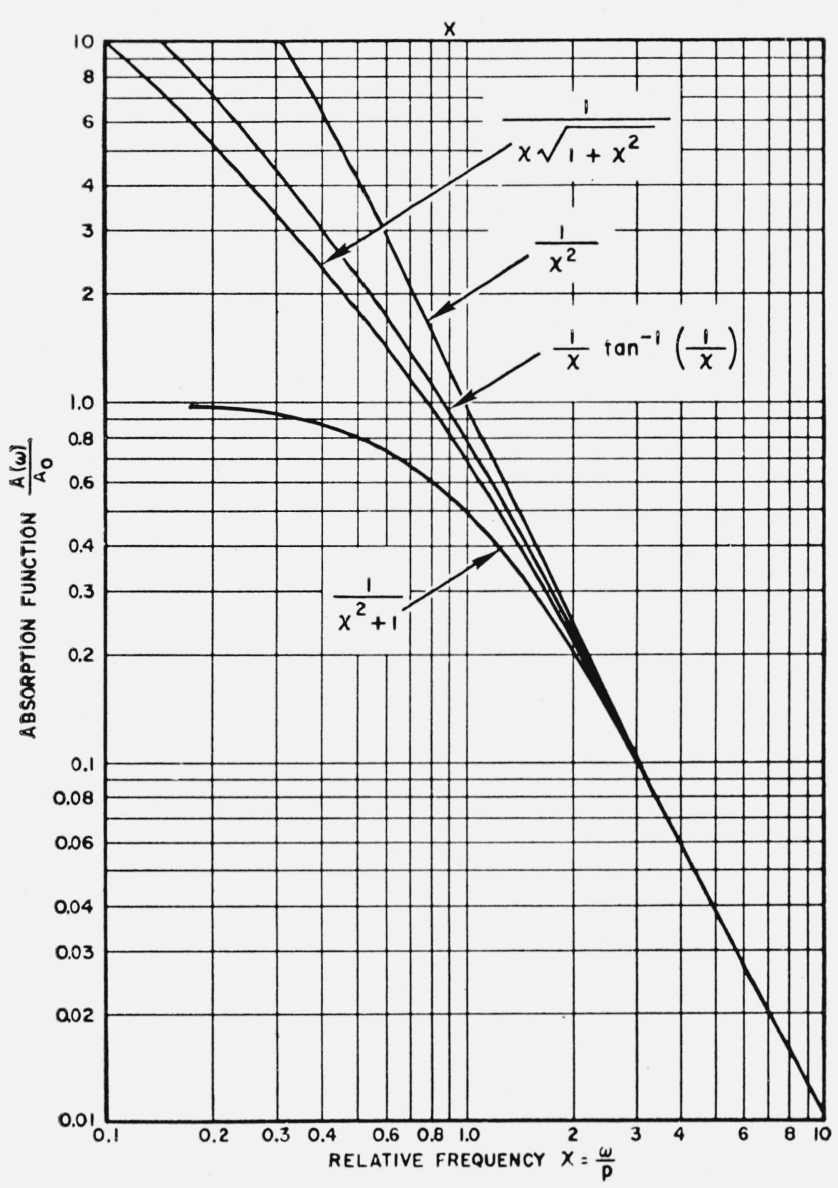

Figure 2. Plots of various absorption functions all of which have the same high frequency behavior. $\omega^{-2}$

apply to the low frequency end of the carrier spectrum where this behavior must eventually change as one approaches the critical frequency of the absorbing layers, since below this plasma cutoff, one has no transmission to be absorbed. In point of fact, it is the high frequency asymptotic behavior of the absorption integral (3.1) which is expected to correspond to the simplified classical form (4.1). We therefore look for other models of the absorption factor $A(\omega)$ which vary as $\omega^{-2}$ for large values of the argument but which have more reasonable variations for small $\omega$.

B. Example 2. The following function removes the low frequency divergence of example (4.1), but in a particular way which controls the solution.

$$
A(\omega)=\frac{A_{0}}{\omega^{2}+p^{2}}
$$

Taking this together with (3.4) gives

$$
\begin{aligned}
N(y) & =\frac{1}{\nu} \frac{d \nu}{d y} \frac{1}{\pi} \int_{0}^{\infty} d x \cos (x \nu) A_{0} \mathcal{L}^{-1}\left(\frac{\omega}{\omega^{2}+p^{2}}\right)_{x} \\
& =A_{0} \frac{1}{\nu} \frac{d \nu}{d y} \frac{1}{\pi} \int_{0}^{\infty} d x \cos (x \nu) \cos (x p)
\end{aligned}
$$
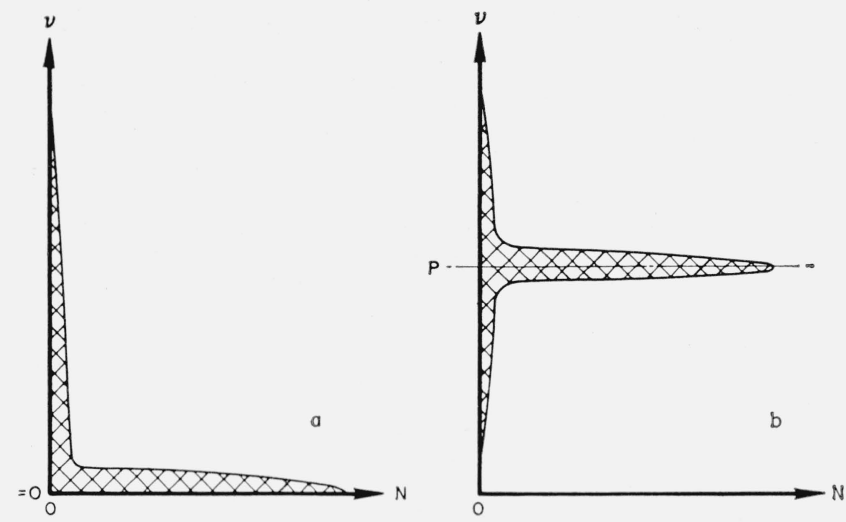

Figure 3. N versus $\nu$ relationships corresponding to two simple models for the radiofrequency variation of the absorption factor: (a) $\mathrm{A}=\mathrm{A}_{0} \omega^{-2}$, and (b) $\mathrm{A}=\mathrm{A}_{0}\left(\omega^{2}+\mathrm{p}^{2}\right)^{-1}$.

or

$$
N(y)=A_{0} \frac{1}{\nu} \frac{d \nu}{d y} \delta(\nu-p)
$$

This tells one that the consequence of (4.3) is that the electron density must be zero everywhere except at the height corresponding to the value $\nu=p$, where $N$ must be infinitely large. This behavoir is shown in figure $3 \mathrm{~b}$, and represents an unrealistic solution for the ionosphere.

C. Example 3. Another model for the absorption factor which preserves the asymptotic factor behavior $A(\omega) \simeq \omega^{-2}$ for high frequencies,

$$
A(\omega)=\frac{A_{0}}{\omega p} \tan ^{-1}\left(\frac{p}{\omega}\right)
$$

is also plotted in figure 2. The Laplace inverse of this function times $\omega$ gives:

$$
\begin{aligned}
N(\nu) & =\frac{1}{\nu} \frac{d \nu}{d y} \frac{1}{\pi} \int_{0}^{\infty} d x \cos (x \nu) \frac{A_{0}}{p} \mathcal{L}^{-1}\left[\tan ^{-1}\left(\frac{p}{\omega}\right)\right]_{x} \\
& =\frac{1}{\nu} \frac{d \nu}{d y} \frac{1}{\pi} \int_{0}^{\infty} d x \cos (x \nu) A_{0} \frac{\sin x p}{x p}
\end{aligned}
$$

or

$$
N(\nu)=\frac{1}{\nu} \frac{d \nu}{d y} \frac{A_{0}}{p}\left\{\begin{array}{l}
1 / 2, \nu<p \\
1 / 4, \nu=p \\
0, \nu>p .
\end{array}\right.
$$

The result represents an electron density model which is zero below the height corresponding to a collision frequency $p=\nu$, and constant above this height. This $N$ versus $\nu$ relation is shown in figure $4 \mathrm{a}$, and the corresponding $N$ versus $y$ plot for an exponential collision (atmosphere) profile $\nu=\nu_{0}$ $\exp -y / H$ is given in figure $4 b$, using the fact that

$$
\frac{1}{\nu} \frac{d \nu}{d y}=-\frac{1}{H}
$$

On the other hand, this model represents a relatively 

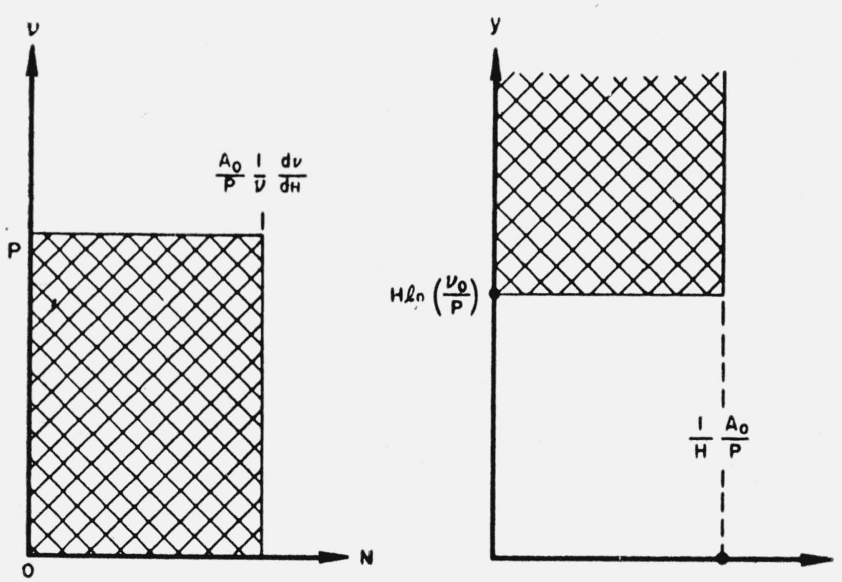

Figure 4. $\mathrm{N}$ versus $\nu$ relationship corresponding to $\mathrm{A}(\omega)=\mathrm{A}_{0}$ $\omega \mathrm{p} \tan ^{-1}(\mathrm{p} / \omega)$ and equivalent electron density profile for an exponentially decreasing collision frequency profile.

idealized electron density profile and serves to point up the profound effect of the low frequency portion of the absorption factor $A(\omega)$.

D. Example 4. A fourth example which also preserves the $\omega^{-2}$ asymptotic behavior

$$
A(\omega)=\frac{A_{0}}{\omega \sqrt{\omega^{2}+p^{2}}}
$$

is plotted in figure 1. The corresponding electron density profile is given by

$$
\begin{aligned}
N(y) & =\frac{1}{\nu} \frac{d \nu}{d y} \frac{1}{\pi} \int_{0}^{\infty} d x \cos (x \nu) A_{0} \mathcal{L}^{-1}\left(\frac{1}{\sqrt{\omega^{2}+p^{2}}}\right) \\
& =\frac{1}{\nu} \frac{d \nu}{d y} \frac{1}{\pi} \int_{0}^{\infty} d x \cos (x \nu) A_{0} J_{0}(x p)
\end{aligned}
$$

or

$$
N(y)=\frac{1}{\nu} \frac{d \nu}{d y} \frac{A_{0}}{\pi}\left\{\begin{array}{cc}
0 & \nu>p \\
\frac{1}{\sqrt{p^{2}-\nu^{2}}} & \nu<p .
\end{array}\right.
$$

This model also represents an electron density which vanishes below a height corresponding to $\nu=p$, but has a more interesting variation above there, as shown in figure 5a. The corresponding $N$ versus $y$ profile for an exponential collision frequency profile is shown in figure $5 \mathrm{~b}$. The plots again show the extreme sensitivity of the electron density profile to the detailed behavior of the absorption function at the low frequency end.

\section{Alternate Solution}

The fundamental solution (3.8) of the absorption integral (3.1) can be simplified in an important way. If we again neglect the gyrofrequency terms, we have

$$
N(y)=\frac{1}{\nu} \frac{d \nu}{d y} \frac{1}{\pi} \int_{0}^{\infty} d x \cos (x \nu) \mathcal{L}^{-1}[\omega A(\omega)]_{x} .
$$

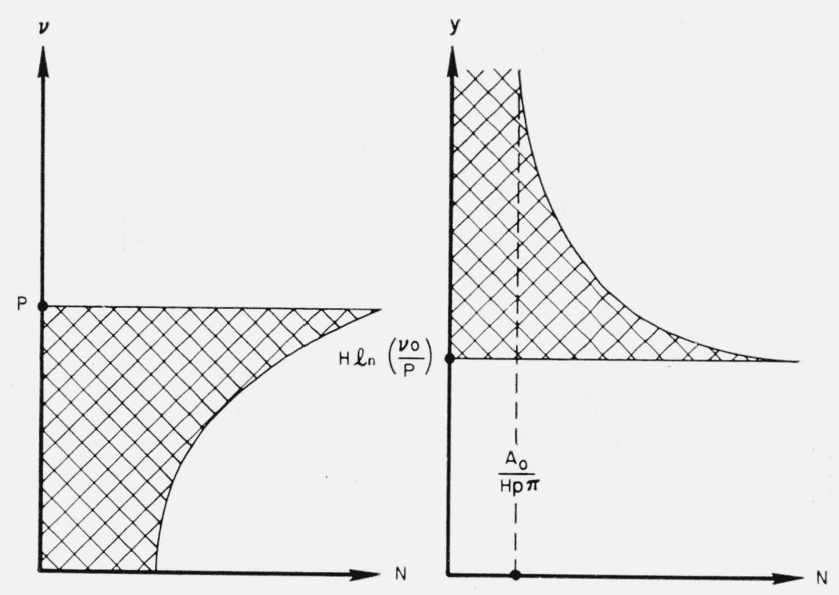

FIgURE 5. $\mathrm{N}$ versus $\nu$ relationship corresponding to $\mathrm{A}=\mathrm{A}_{0} /$ $\omega \sqrt{\omega^{2}}+\mathrm{p}^{2}$ and equivalent electron density for an exponential collision frequency profile.

Further reduction is achieved by carrying out the cosine transform on the inverse Laplace operation in a way which is independent of the function $\omega A(\omega)$. To do so, we use the usual complex representation for the cosine function and introduce an infinitesimal attenuation term in the $x$ integration.

$$
\begin{array}{r}
N(y)=\frac{1}{\nu} \frac{d \nu}{d y} \lim _{\epsilon \rightarrow 0} \frac{1}{\pi} \int_{0}^{\infty} d x e^{-\epsilon x}\left(\frac{e^{i x_{\nu}}+e^{-i x \nu}}{2}\right) \mathcal{L}^{-1}[\omega A(\omega)]_{x} \\
=\frac{1}{\nu} \frac{d \nu}{d y} \frac{1}{2 \pi} \lim _{\epsilon \rightarrow 0} \int_{0}^{\infty} d x\left[e^{-x(\epsilon-i \nu)}\right. \\
\left.+e^{-x(\epsilon+i \nu)}\right] \mathcal{L}^{-1}[\omega A(\omega)]_{x} .
\end{array}
$$

Cast in this form, one sees that the integration has the effect of undoing the inverse Laplace transform and simply to evaluate the function at those values given by the coefficients of $x$ in the exponential factors,

$N(y)=\frac{1}{\nu} \frac{d \nu}{d y} \lim _{\epsilon \rightarrow 0} \frac{1}{2 \pi}\left\{[\omega A(\omega)]_{\epsilon-i \nu}+[\omega A(\omega)]_{\epsilon+i \nu}\right\}$.

The expression provides an alternative solution to the basic absorption (3.1), and could have been derived more directly by contour integration techniques.

The remarkable feature of our alternate solution (5.3) is that it depends on the value of the absorption function $A(\omega)$ only at the complex points $\epsilon \pm i \nu(y)$, where $y$ is the altitude at which $N$ is to be computed. However, one is reminded that the absorption function is measured by experiments only for real positive values of the carrier frequency $\omega$. To exploit the new solution (5.3), one must use analytic continuation from the real positive axis to the complex points $\epsilon \pm i \nu$, as shown in figure 6 . In general, this analytic continuation will be determined by all measured values of $A(\omega)$. However, figure 6 suggests that the values at $\epsilon \pm i \nu$ will be especially sensitive to values of $A(\omega)$ near the origin, and this low frequency sensitivity of the inversion process has already been noted empirically in the examples of section 4 . We 
$\omega$ PLANE

- $\epsilon+\mathrm{i \nu}$

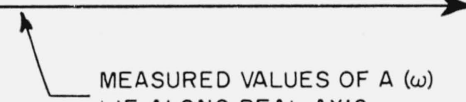

- $\epsilon-i \nu$

Figure 6. Complex frequency plane showing points at which absorption function is to be evaluated and emphasizing that measured values all lie along positive real axis.

thus have a more succinct demonstration of the limitation of the nondeviative solution considered here and suggests the more difficult problem of deviative absorption which will recognize the behavior of the absorption-reflection process at low frequencies.

As a concluding exercise, it may be useful to resolve the first example of section 4 with the alternate solution. If we again take $A(\omega)=A_{0} \omega^{-2}$, solution (5.3) gives

$$
\begin{aligned}
N(y) & =\frac{1}{\nu} \frac{d \nu}{d y} \lim _{\epsilon \rightarrow 0} \frac{A_{0}}{2 \pi}\left(\frac{1}{\epsilon-i \nu}+\frac{1}{\epsilon+i \nu}\right) \\
& =\frac{1}{\nu} \frac{d \nu}{d y} \frac{A_{0}}{\pi} \lim _{\epsilon \rightarrow 0}\left(\frac{\epsilon}{\epsilon^{2}+\nu^{2}}\right) \\
& =\frac{1}{\nu} \frac{d \nu}{d y} A_{0} \delta(\nu-0)
\end{aligned}
$$

where we have used here the limit definition of the Dirac delta function. Of course, this solution reproduces the earlier result (4.2) as one would expect.

This problem was suggested by some experimental work of G. C. Little and his colleagues, which is now in progress. Its solution benefited from conversations with James R. Wait and M. L. Goldberger.

\section{References}

Appleton, E. V. (1937), Regularities and irregularities in the ionosphere, Proc. Roy. Soc. (London) A169, 451.

Best, J. E., and Ratcliffe, J. A. (March 1938), Diurnal variation of ionospheric absorption of radio waves, Proc. Phys. Soc. 50, 233.

Beynon, W. J. G., and Davies, K. (1954), A study of vertical incidence ionospheric absorption at two megacycles, Physies of the Ionosphere, report of 1954 Cambridge Conference (Physical Society London), 40.

Chapman, S. (1931), The absorption and dissociative or ionizing effect of monochromatic radiation in an atmosphere on a rotating earth, Proc. Phys. Soc. 43, 26.

Chapman, S., and Davies, K. (1958), On the approximate daytime constancy of the absorption of radio waves in the lower ionosphere, J. of Atmospheric and Terrest. Phys. 13, 86 .

Davies, K, and Hago, E. L. (1955), Ionospheric absorption at Prince Rupert, J. of Atmospheric and Terrest. Phys. 6, 18 .

Farmer, F. T., and Ratcliffe, J. A. (1935), Measurements of the absorption of wireless waves in the ionosphere, Proc. Roy. Soc. (London) A151, 370 .

Jaeger, J. C. (1947), Equivalent path and absorption in an ionospheric region, Proc. Phys. Soc. 59, 87.

Little, G. C. (1961), Private communication regarding current experiments at the National Bureau of Standards and the University of Alaska.

Ratcliffe, J. A. (1959), The magnetionic theory and its applications to the ionosphere (Cambridge University Press).

Sen, H. K., and Wyller, A. A. (1960), On the generalization of the Appleton-Hartree magnetionic formulas, J. Geophys. Res. 65, 3931 . 